\title{
Comparative proteogenomic analysis of right-sided colon cancer, left-sided colon cancer and rectal cancer reveals distinct mutational profiles
}

\author{
Robin Imperial ${ }^{1}$, Zaheer Ahmed', Omer M. Toor ${ }^{1,2}$, Cihat Erdoğan ${ }^{3}$, Ateeq Khaliq², Paul Case ${ }^{2}$, James Case ${ }^{4}$, \\ Kevin Kennedy ${ }^{5}$, Lee S. Cummings ${ }^{6}$, Niklas Melton ${ }^{7}$, Shahzad Raza ${ }^{1,2}$, Banu Diri ${ }^{8}$, Ramzi Mohammad ${ }^{9}$, \\ Bassel El-Rayes ${ }^{10}$, Timothy Pluard ${ }^{1,2}$, Arif Hussain ${ }^{11,12}$, Janakiraman Subramanian ${ }^{1,2^{*}}$ and Ashiq Masood ${ }^{13^{*}}$
}

\begin{abstract}
Right-sided colon cancer (RCC) has worse prognosis compared to left-sided colon cancer (LCC) and rectal cancer. The reason for this difference in outcomes is not well understood. We performed comparative somatic and proteomic analyses of RCC, LCC and rectal cancers to understand the unique molecular features of each tumor sub-types. Utilizing a novel in silico clonal evolution algorithm, we identified common tumor-initiating events involving APC, KRAS and TP53 genes in RCC, LCC and rectal cancers. However, the individual role-played by each event, their order in tumor development and selection of downstream somatic alterations were distinct in all three anatomical locations. Some similarities were noted between LCC and rectal cancer. Hotspot mutation analysis identified a nonsense mutation, APC R1450* specific to RCC. In addition, we discovered new significantly mutated genes at each tumor location, Further in silico proteomic analysis, developed by our group, found distinct central or hub proteins with unique interactomes among each location. Our study revealed significant differences between RCC, LCC and rectal cancers not only at somatic but also at proteomic level that may have therapeutic relevance in these highly complex and heterogeneous tumors.
\end{abstract}

Keywords: Right-sided colon cancer, Left-sided colon cancer, Rectal cancers, Clonal evolution, Proteomics, Hotspot mutations

Often grouped as one disease, right-sided colon cancer (RCC, originating from cecum, ascending colon, hepatic flexure) and left-sided colon cancer (LCC, originating from splenic flexure, descending colon, sigmoid colon) represent clinically distinct entities with significant differences in their prognosis and treatment outcomes [1, 2]. Therefore, given their anatomic continuity, the reason for these clinical differences presumably lie at the molecular level delineated by embryological origin. Previous studies have sought to identify these differences by

\footnotetext{
* Correspondence: jsubramanian@saint-lukes.org; ashiq_masood@rush.edu ${ }^{1}$ Department of Medicine, University of Missouri-Kansas City School of Medicine, Kansas City, MO 64108, USA

${ }^{13}$ Division of Hematology/Oncology and Cell Therapy, Rush University Medical Center, Chicago, IL 60612, USA

Full list of author information is available at the end of the article
}

analyzing significantly mutated genes and RNA expression [3, 4]. However, molecular differences including significant specific amino acid alterations (hot spots), proteomic differences and order of mutations in clonal evolution of these tumors have not been studied. We used somatic and proteomic data of colorectal cancers from The Cancer Genome Atlas (TCGA) $[4,5]$, Memorial Sloan Kettering Cancer Center (MSKCC) [6] and The Cancer Proteome Atlas (TCPA) [7] to study proteogenomic differences in these tumors (See Additional files 1 and 2).

\section{Results and discussion Clonal evolution trajectories \\ Understanding the mutational timing and evolutionary trajectory of tumors is key to investigate the molecular}

(c) The Author(s). 2018 Open Access This article is distributed under the terms of the Creative Commons Attribution 4.0 International License (http://creativecommons.org/licenses/by/4.0/), which permits unrestricted use, distribution, and 


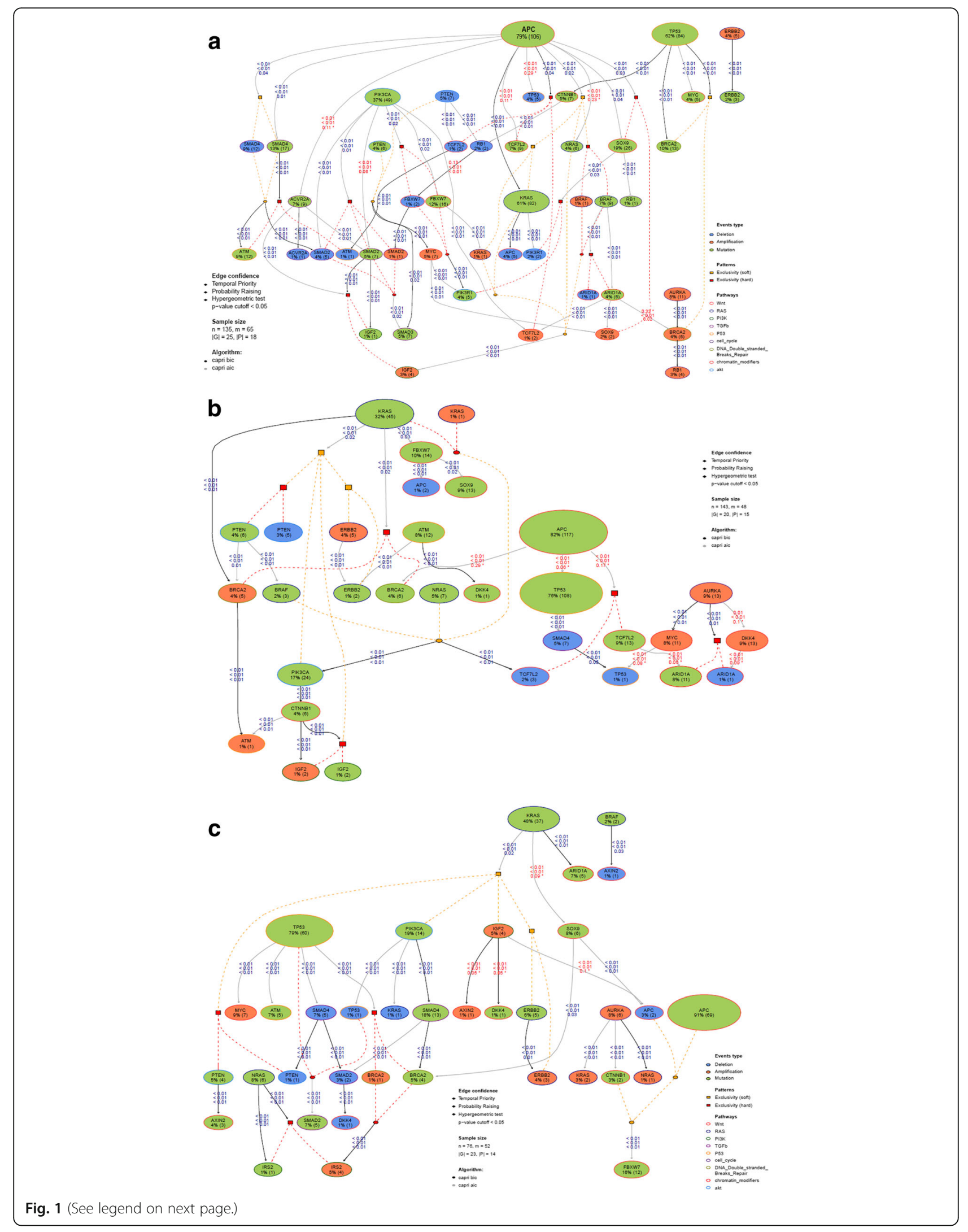


(See figure on previous page.)

Fig. 1 shows ensemble-level clonal evolution trajectories in colorectal cancer using CAPRI algorithm. The events of the model are connected by dashed lines where red dotted lines denote hard and orange denotes soft exclusivity. Algorithm uses both Bayesian information criterion 'BIC' and Akaike information criterion 'AIC' as a regularization. Non-parametric bootstrap scores (NPB) are shown in the figure with hypergeometric test $p$-value cutoff of $<0.05$. Other relations including temporal priority, probability raising are shown in Fig. $1 \mathrm{a}, \mathrm{b}$, and $\mathrm{c}$ and reported data in Additional file 4. 1a) clonal evolution in RCC, 1b) clonal evolution in LCC and 1c) clonal evolution in rectal cancers

underpinnings of cancer development and progression. Thus, we applied the PiCnIc (Pipeline for Cancer Inference) algorithm to our data to study ensemble-level cancer progression models and predict the evolutionary mutational trajectories between RCC, LCC and rectal cancers in the TCGA cohort (see Additional file 3). All three cancer locations had mutations in APC, TP53 and KRAS, possibly reflecting common initiating somatic events (Fig. 1). However, there were differences in the hierarchical groupings of mutations that surrounded these events.

In RCC ( $n=135$; Fig. 1a; Additional file 4: Table S1), APC somatic mutations and TP53 somatic mutations were independent events. APC somatic mutations 'selected' for KRAS mutations or amplifications. APC somatic mutations also 'selected' for SMAD4 somatic mutations or deletions, BRAF mutations and amplification. KRAS and BRAF showed mutual exclusivity. Interestingly, alterations in FBWX7, TCF7L2, and SMAD2 clustered in RCC tumors harboring APC and PIK3CA mutations. With respect to TP53, alterations in this location were associated with CTNNB1, MYC or/and BRCA2 mutations.

In LCC $(n=143$; Fig. 1b; Additional file 4: Table S2), KRAS somatic mutations 'selected' for BRCA2 amplification, PTEN deletions or somatic mutations, PIK3CA somatic mutations, IGF2 amplification or somatic mutations and ERBB2 amplification or somatic mutations. Unlike RCC, alterations in PIK3CA were a late event in LCC and IGF2 amplification via CTNNB1. APC seemed to 'select' for TP53, but this did not reach statistical significance $(p=0.06)$. Similarly, APC somatic mutations 'selected' for BRCA2 mutations and TCF7L2 somatic mutations or deletions, but this association also did not reach statistical significance ( $p=0.3$ and $p=0.2$, respectively).

In rectal cancers ( $n=76$; Fig. $1 c$; Additional file 4: Table S3), key initial mutations are split between TP53 and KRAS. TP53 'selects' for MYC amplification, SMAD4 deletion and BRCA2 somatic mutation or amplification. KRAS 'selects' for PTEN deletion or somatic mutations, PIK3CA somatic mutations, IGF2 amplification and ERBB2 amplification or somatic mutations. Among rectal cancer patients with AURKA mutations there is clustering of NRAS amplifications.

Our model shows significant differences in the mutational profiles of genes between RCC and LCC; the early common somatic gene mutations are associated with the 'selection' of different subsequent genomic events in RCC compared to LCC. Our results suggest that although LCC and rectal cancers have some similarities in the tumor progression model wherein KRAS 'selected' for several genes in common (such as PIK3CA, IGF2, and ERBB2 alterations), significant differences were also noted between these two sites. Taken altogether, our results show non-adherence to the established Vogelstein linear progression model of colorectal cancer progression from normal mucosa to adenoma to carcinoma [8]. Further, our data suggest that RCC, LCC and rectal cancers have distinct mutational behavior in the context of their evolutionary trajectories, mutational timing during cancer development and progression. However, initial events such as mutation in the gatekeeper gene, APC, appear to be similar in colorectal cancers irrespective of location.

\section{Mutation hotspot analysis}

We studied somatic mutations at the residue sites that can disrupt functional protein domains leading to tumorigenesis and clonal evolution via selective pressure (see Methods in Additional file 3). We found APC $\mathrm{R} 1450 *$ to be a significant mutation specifically enriched in RCC (12-15\%) compared to LCC (1\%) and rectal tumors (1\%) in both the TCGA and MSKCC datasets (all $p<0.001$, Fig. 2a). To our knowledge, this is the first report to describe the APC R1450* mutation as being predominantly located in RCC. This particular hotspot in APC is exclusively a truncation mutation and lies within the MCR domain (residues 1282-1581; [9]) of the protein, which is a highly mutated area. The resulting truncated mutant conserves beta-catenin binding sites (15 AA repeats) but loses all three axin-binding sites (SAMP repeats) and microtubule interaction via EB1 and PdZ domains. Unlike APC R1450*, the frequency of other mutations within this region is relatively similar among the TCGA and MSKCC data sets. The relative frequencies of non-R1450* mutations within the MCR domain of APC for RCC were 63 and $64 \%$ in the TCGA and MSKCC data sets, respectively, for LCC 52 and 51\%, respectively, and for rectal cancers $64 \%$ vs $58 \%$ (which did not meet statistical significance, $p=0.35$ ). APC R1450* mutations are mutually exclusive from $\beta$-catenin destruction complex genes suggesting that they may be 


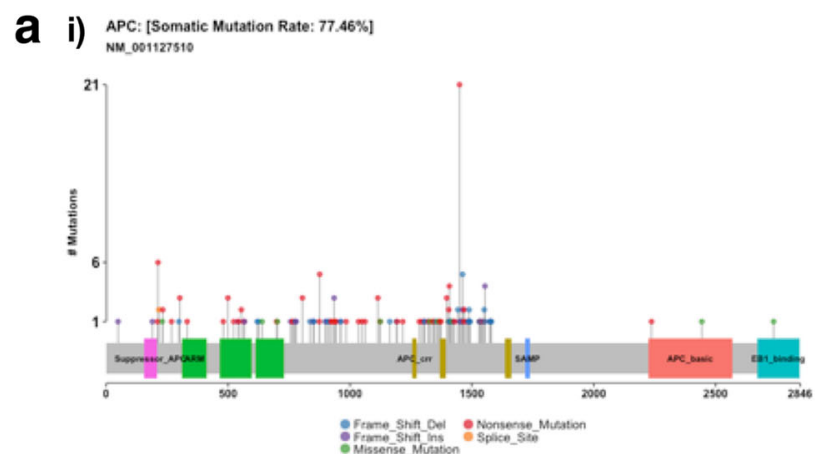

APC: [Somatic Mutation Rate: 66.67\%]

NM_.001127510

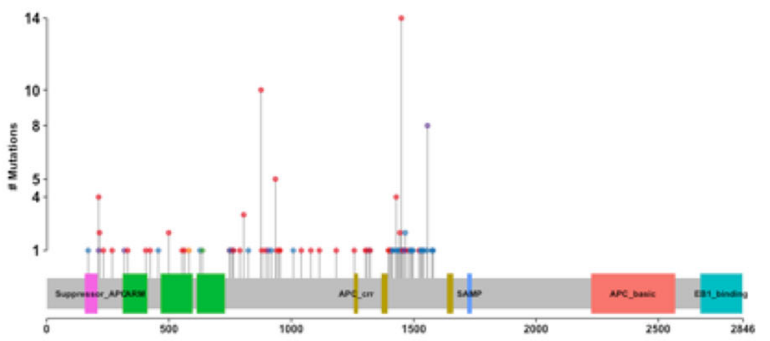

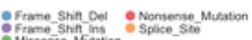

APC: [Somatic Mutation Rate: $82.54 \%$ ]

NM. 001127510

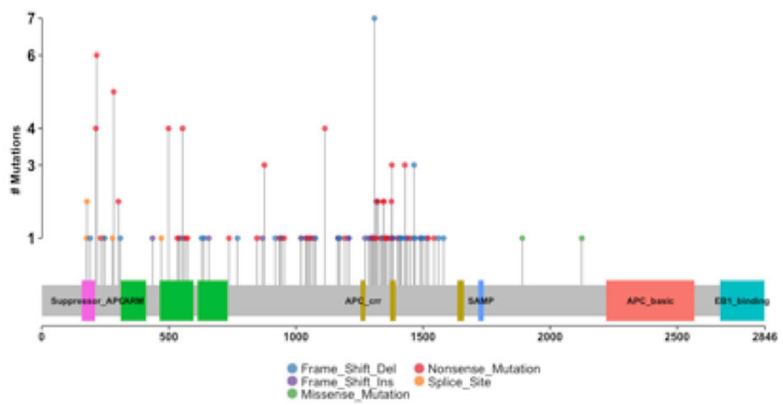

APC: [somatic Mutation Rate: $56.08 \%$ ]

NM_ 001127510

iii) APC: [Somatic Mutation Rate: 80.9\%]
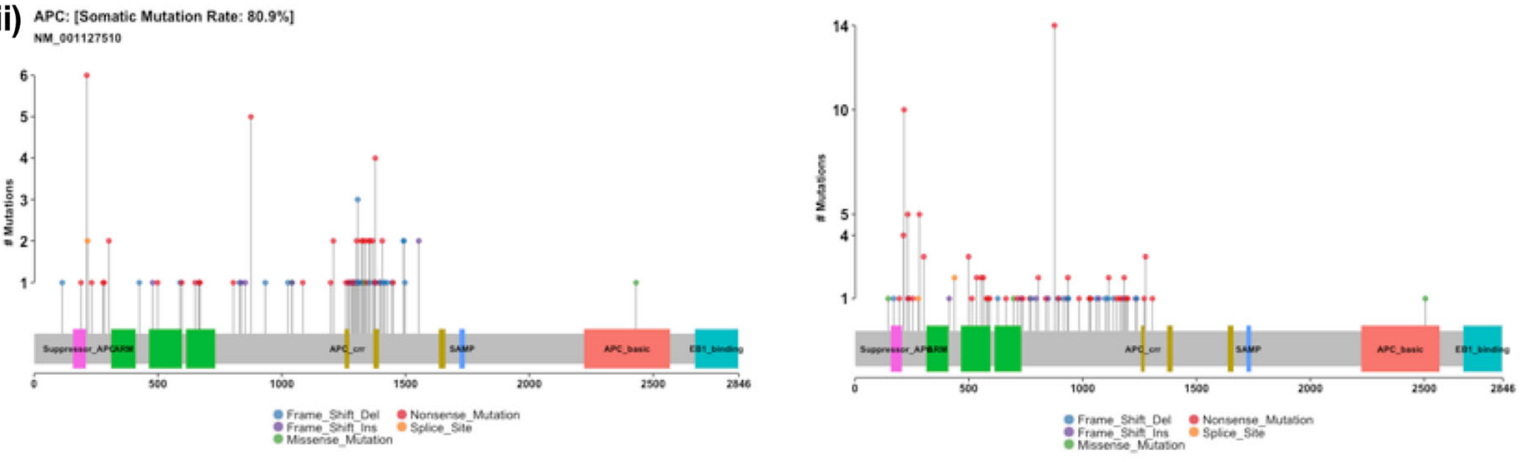

b

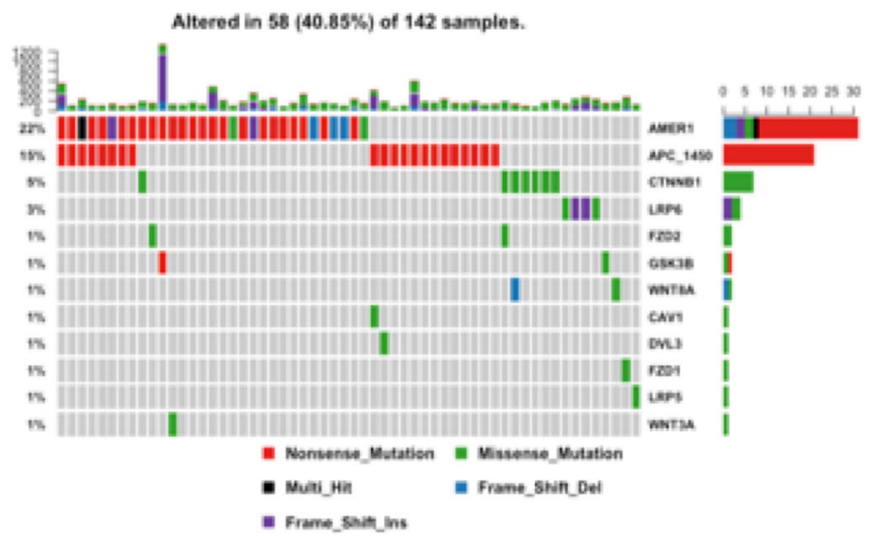

Fig. 2 a shows the frequency of APC hotspot the R1450 residue in (i) right-sided colon cancers, (ii) left-sided colon cancers and (iii) rectal cancers in TCGA (left) and MSKCC (right) datasets. Y-axis represent total number of mutations at each residue. b shows the mutual exclusivity of APC R1450* (APC_1450) compared to other genes of $\beta$-Catenin destruction complex in RCC. "APC_MCR" represents other APC mutations within the MCR region that are not at the 1450 residue. The bar plot above the oncoplot represents total mutations in each sample 
A

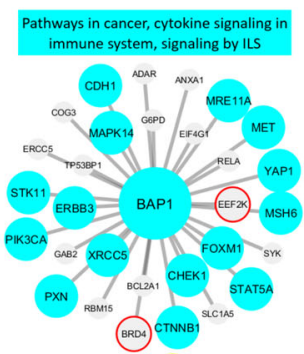

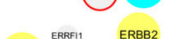

MSH2 2 ERPFII ERBB82

(2an) icFir

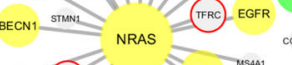

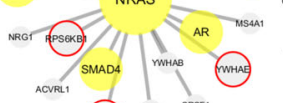

ncrer sMAD4

ERBB signaling pathwway, sigraling by
ERBB2, GRB2 events in ERBB2 signaling

B

$$
\begin{gathered}
\text { Pathways in cancer, } \\
\text { prostate cancer }
\end{gathered}
$$

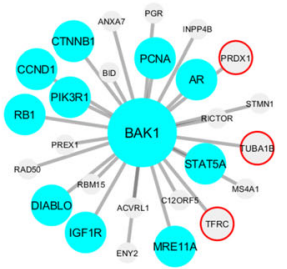

(18)
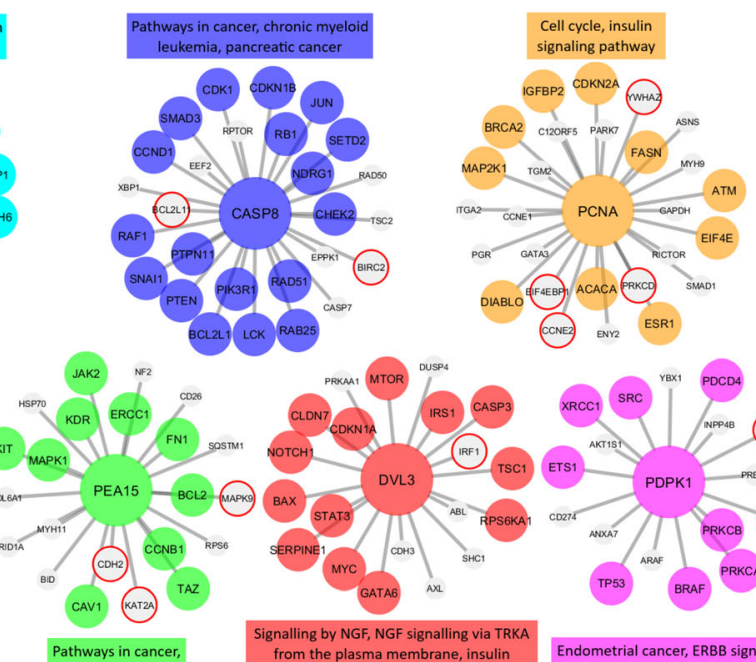

Prem, MTOR Dusers

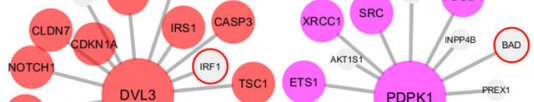

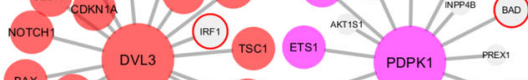

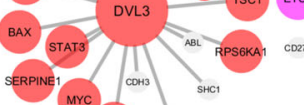

(atrate

TPS3

Pathways in cancer,
focal adhesion

Signalling by NGF, NGF signalling via TRKA

fom the plasma membrane, insulin Endometrial cancer, ERBB signaling receptor signalling cascade

pathway, non small cell lung cancer

ERBB signaling pathway, NGF signalling via TRKA
from the plasma membrane, signaling by ERBBA
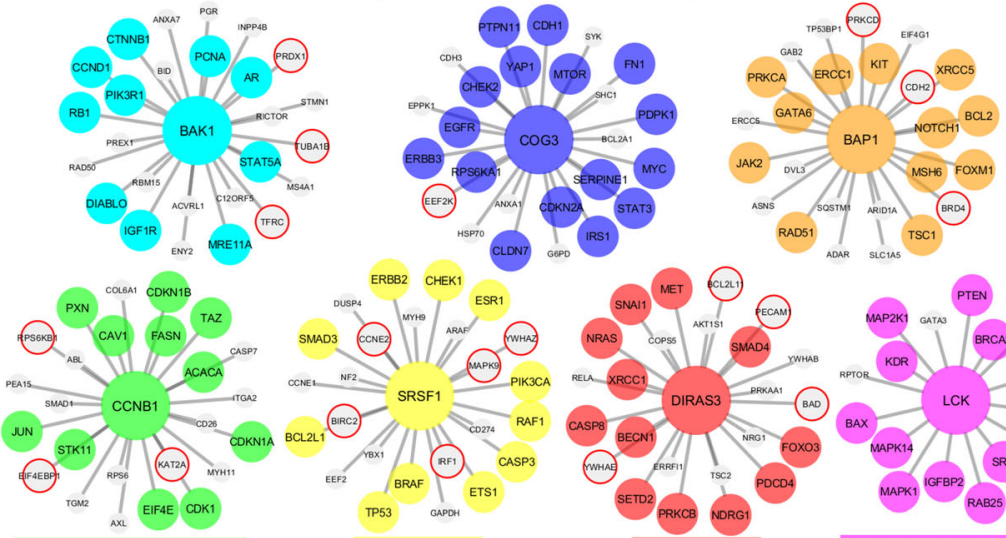

ERBB2 CHEK1 ESR1

-

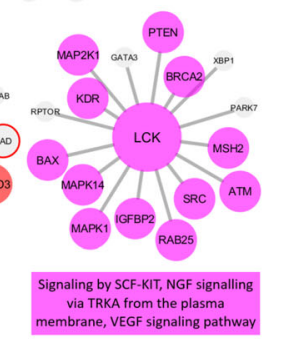

C
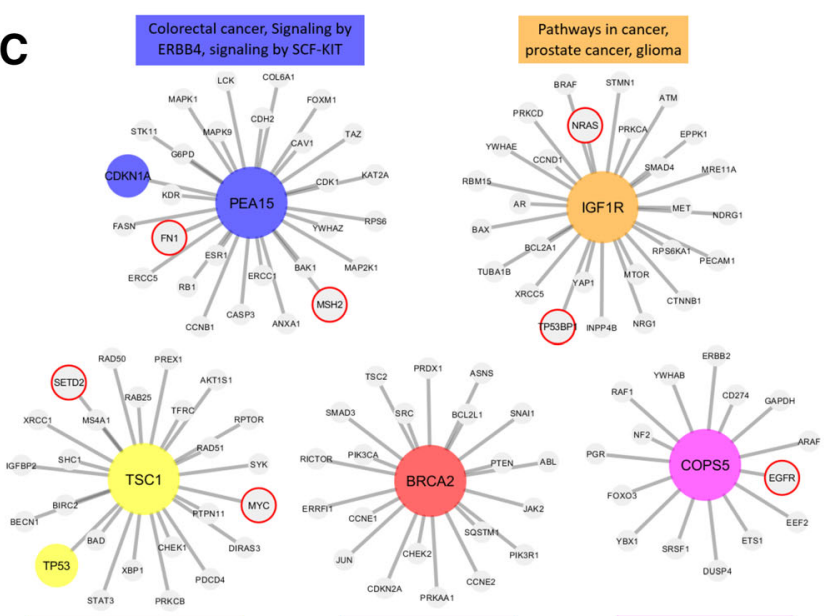

Pathways in cancer, chronic

myeloid leukemia, signaling by
the B-cell receptor BCR

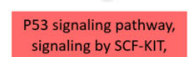

Endometrial cancer, non small cell lung cancer,
signaling by ERBB4

Fig. 3 (See legend on next page.) 
(See figure on previous page.)

Fig. 3 shows the hub genes and neighbors in the disease-related sub-networks obtained by the most successful KDE method (in terms of precision score) in a RCC, $\mathbf{b}$ LCC and $\mathbf{c}$ rectal cancers. The genes registered in DisGeNET and experimentally confirmed for the diseases are shown with colored and larger nodes. Among these, genes that are not colored but have a red frame have a PMID value of one (e.g. have one supporting publication). There is no entry in DisGeNET for the grey colored nodes. Also, the most associated top three biological pathways, to which each module is related, are given above or below the relevant module to annotate each module

early events in right-sided colon cancer tumorigenesis (Fig. 2b). Given the recent findings by Zhang et al. demonstrating the efficacy of TASIN-1(small molecule inhibitor) in a murine xenograft model of human colorectal cancer harboring a truncation mutation (A1309*) similar to APC R1450* suggests that this mutation may be viable therapeutic target [10]. In addition, we performed significantly mutated gene analysis and discovered newer driver genes at each location (see Additional file 5: Figure S1).

\section{Proteogenomic analysis}

Using The Cancer Proteome Atlas (TCPA) data, we examined RCC, LCC and rectal cancers by proteomic cancer co-expression subnetworks using association estimators methodology previously described by our group (see Methods in Additional file 3). Interestingly, no common protein emerged as having a centralized role (hub protein) across all 3 cancer locations. Within protein-protein interaction networks, several hub proteins, and their respective interactomes, were found to be unique to each of the locations (Fig. 3, see Additional file 6: Table S4).

Several hub proteins that might have a major role in RCC were identified: BAP1 (tumor suppressor gene) CASP8 (apoptosis) PCNA (DNA repair) NRAS (RTKRAS pathway) PEA15 (apoptosis and RET signaling) DVL3 (cell proliferation and ATM-dependent DNA damage response) and PDPK1 (growth regulation) (Fig. 3a). The potentially significant hub proteins in LCC were: BAP1, BAK1 (apoptosis and prognostic in breast cancer) COG3 (protein glycosylation/golgi function) CCNB1 (mitosis and prognosis in breast cancer) SRSF1 (RNA splicing and prognosis in small cell lung cancer) DIRAS3 (tumor suppressor gene) and LCK (resistance to apoptosis) (Fig. 3b). Hub proteins unique to rectal cancers were: IGF1R (proliferation, invasion, migration), TSC1 (cell growth) BRCA2 (DNA repair) and COPS5 (multiple pathways) (Fig. 3c).

BAP1 was found to have a prominent role in both RCC and LCC. Although there are several conserved interactions, the BAP1 interactome of LCC diverges from that of RCC. Among the conserved interacting proteins are: BRD4, ADAR, GAB2, SLC1A5, EIF4G1, ERCC5 and TP53BP1, BRD4, ADAR, MSH6, FOXM1 and XRCC5. Specific to LCC, BAP1 showed interactions with ERCC1,
PRKCA, GATA6, JAK2, RAD51, TSC1, RSC1, NOTCH1, BCL2, KIT, PRKCD, CDH2, ARID1A, ASNS, SQSTM1 and DVL3. Specific to RCC, BAP1 was noted to interact with CDH1, MAPK14, MRE11A, MET, YAP1, STK11, ERBB3, PIK3CA, PXN, CHEK1, CTNNB1, STAT5A, EEF2K, G6PD, COG3, RBM15, BCL2A1, SYK, RELA and ANXA1.

Our results suggest BAP1 may have an essential role in carcinogenesis of colon cancer with conserved as well as divergent evolutionary interactions with other proteins in RCC and LCC that are largely absent in rectal cancers.

A somewhat surprising observation from this analysis is that the protein hubs and their interactomes are distinct for each of the anatomically defined tumor sites examined. Further, these protein signatures are not necessarily concordant with the somatic tumor profiles. Identifying alterations in tumor DNA and RNA have been of paramount importance. Clarifying post-transcriptional events and protein-protein interactions will also be highly relevant to understanding the variations in tumor biology and clinical behavior of these tumors. Prospective studies are needed to validate our findings and their implications in the clinical outcomes.

\section{Additional files}

Additional file 1: Patients demographics from TCGA. (DOCX $19 \mathrm{~kb}$ )

Additional file 2: Inclusion and exclusion criteria for somatic mutation analysis. MSI-H, POLE mutation samples, rectosigmoid and transverse colon cancers were excluded for analysis(highlighted green). (TIF $666 \mathrm{~kb}$ )

Additional file 3: Methods section. (DOCX $52.9 \mathrm{~kb}$ )

Additional file 4: Tables S1-S3. PicNiC statistics (bic and aic) for RCC, LCC and rectal cancers. (XLSX $43 \mathrm{~kb}$ )

Additional file 5: Somatic mutation analysis for RCC, LCC and rectal cancers. (DOCX $433 \mathrm{~kb}$ )

Additional file 6: Table S4. Proteomics pathway and gene level analysis results. (XLSX $47 \mathrm{~kb})$

\section{Abbreviations}

AIC: Akaike information criterion; BIC: Bayesian information criterion; CAPRI: Cancer progression inference algorithm; KDE: Kernel density estimator; LCC: Left colon cancer; MSKCC: Memorial sloan kettering cancer center; PiCnlc: Pipeline for cancer inference; PMID: Pubmed identifier; RCC: Right colon cancer; TCGA: The cancer genome atlas; TCPA: The cancer proteome atlas

\section{Acknowledgements}

Part of A.H.'s time was supported by a Merit Review Award (I01 BX000545), Medical Research Service, Department of Veterans Affairs. 


\section{Funding}

Not applicable.

\section{Availability of data and materials}

TCGA somatic mutation data for colorectal cancers can be obtained from the GDC legacy archive and Broad GDAC Firehose. Proteomic data can be downloaded from The Cancer Proteome Atlas (TCPA). The datasets used and analyzed in the current study are also available from the corresponding author in response to reasonable requests.

\section{Authors' contributions}

$\mathrm{RI}$ and $\mathrm{AM}$ contributed to the design of study, involved in acquisition, analysis and interpretation of data; involved in drafting the manuscript and revising it critically for important intellectual content. ZA, OMT, CE, AK, PC, JC, KK, NM, BD involved in data acquisition, analysis and interpretation of data and involved in drafting the manuscript. LSC, SR, RM, BE, TP, AH, JS were involved in revising the manuscript critically for important intellectual content. All authors read and approved the final manuscript.

\section{Ethics approval and consent to participate}

Not applicable.

\section{Consent for publication}

Not applicable.

\section{Competing interests}

Ashiq Masood Advisory board and speaker Bureau Bristol-Myers Squibb and Boehringer Ingelheim, Honorarium Biocept. Janakiraman Subramanian Advisory board - Astra Zeneca, Pfizer, Boehringer Ingelheim, Alexion, Paradigm, Bristol-Myers Squibb Speakers Bureau - Astra Zeneca, Boehringer Ingelheim, Lilly Research Support - Biocept and Paradigm. Arif Hussain Advisory board - Novartis, Bayer, Astra Zeneca, Consultant - Bristol-Myers-Squibb. All other authors declare that they have no competing interest.

\section{Publisher's Note}

Springer Nature remains neutral with regard to jurisdictional claims in published maps and institutional affiliations.

\section{Author details}

${ }^{1}$ Department of Medicine, University of Missouri-Kansas City School of Medicine, Kansas City, MO 64108, USA. ²Division of Oncology, Saint Luke's Cancer Institute, University of Missouri School of Medicine, 4321 Washington St, Kansas City, MO 64111, USA. ${ }^{3}$ Department of Computer Engineering, Namik Kemal University, Tekirdag, Turkey. ${ }^{4}$ ASPIRE Foundation, Saint Luke's Health System of Kansas City, Kansas City, MO 64111, USA. ${ }^{5}$ Division of Cardiovascular Research, Saint Luke's Hospital, Kansas City, MO 64111, USA. ${ }^{6}$ Department of Surgery, University of Missouri-Kansas City, Kansas City, MO 64108, USA. ${ }^{7}$ Department of Computer Sciences, Missouri University of Science and Technology, Rolla, MO 65409, USA. ${ }^{8}$ Department of Computer Engineering, Yildiz Technical University, Istanbul, Turkey. ${ }^{9}$ Wayne State University, Karmanos Cancer Institute, Detroit, MI 48201, USA. ${ }^{10}$ Department of Hematology and Medical Oncology, Winship Cancer Institute, Emory University, Atlanta, GA 30322, USA. " ${ }^{1}$ Division of Oncology, University of Maryland Greenebaum Comprehensive Cancer Center, Baltimore, MD 20201, USA. ${ }^{12}$ Baltimore Veterans Affairs Medical Center, Baltimore, MD 21201, USA. ${ }^{13}$ Division of Hematology/Oncology and Cell Therapy, Rush University Medical Center, Chicago, IL 60612, USA.

Received: 28 August 2018 Accepted: 30 November 2018 Published online: 21 December 2018

\section{References}

1. Venook AP, Niedzwiecki D, Lenz H-J, et al. CALGB/SWOG 80405: phase III trial of irinotecan/5-FU/leucovorin (FOLFIRI) or oxaliplatin/5-FU/leucovorin (mFOLFOX6) with bevacizumab (BV) or cetuximab (CET) for patients (pts) with KRAS wild-type $(w t)$ untreated metastatic adenocarcinoma of the colon or rectum (MCRC). J Clin Oncol. 2014;32:LBA3. https://doi.org/10.1200/ jco.2014.32.18_suppl.lba3.

2. Hong TS, Clark JW, Haigis KM. Cancers of the Colon and Rectum: identical or fraternal twins? Cancer Discov. 2012;2:117-21. https://doi.org/10.1158/ 2159-8290.CD-11-0315.
3. Hu W, Yang Y, Li X, et al. Multi-omics approach reveals distinct differences in left- and right-sided Colon Cancer. Mol Cancer Res. 2018;16:476-85. https://doi.org/10.1158/1541-7786.MCR-17-0483.

4. Muzny DM, Bainbridge MN, Chang K, et al. Comprehensive molecular characterization of human colon and rectal cancer. Nature. 2012;487:330-7. https://doi.org/10.1038/nature11252.

5. Grossman RL, Heath AP, Ferretti V, et al. Toward a shared vision for Cancer genomic data. N Engl J Med. 2016;375:1109-12. https://doi.org/ 10.1056/NEJMp1607591.

6. Yaeger R, Chatila WK, Lipsyc MD, et al. Clinical sequencing defines the genomic landscape of metastatic colorectal cancer. Cancer Cell. 2018;33: 125-136.e3. https://doi.org/10.1016/j.ccell.2017.12.004.

7. Li J, Lu Y, Akbani R, et al. TCPA: a resource for cancer functional proteomics data. Nat Methods. 2013;10:1046-7. https://doi.org/10.1038/nmeth.2650.

8. Vogelstein B, Papadopoulos N, Velculescu VE, et al. Cancer genome landscapes. Science (80-). 2013;339:1546-58. https://doi.org/10.1126/science.1235122.

9. Miyoshi $\mathrm{Y}$, Nagase $\mathrm{H}$, Ando $\mathrm{H}$, et al. Somatic mutations of the APC gene in colorectal tumors: mutation cluster region in the APC gene. Hum Mol Genet. 1992;1:229-33.

10. Zhang L, Theodoropoulos PC, Eskiocak U, et al. Selective targeting of mutant adenomatous polyposis coli (APC) in colorectal cancer. Sci Transl Med. 2016;8:361ra140. https://doi.org/10.1126/scitranslmed.aaf8127.

\section{Ready to submit your research? Choose BMC and benefit from:}

- fast, convenient online submission

- thorough peer review by experienced researchers in your field

- rapid publication on acceptance

- support for research data, including large and complex data types

- gold Open Access which fosters wider collaboration and increased citations

- maximum visibility for your research: over $100 \mathrm{M}$ website views per year

At $\mathrm{BMC}$, research is always in progress.

Learn more biomedcentral.com/submissions 